\title{
Charmless B Decays at LHCb
}

\author{
Laurence Carson ${ }^{* \dagger}$ \\ University of Glasgow \\ E-mail: l.carson@physics.gla.ac.uk
}

The potential of the LHCb experiment to test the Standard Model and probe New Physics effects by making precision measurements using decays of $B$ hadrons to charmless hadronic final states is explored. The CKM angle $\gamma$ can be measured using NP-sensitive decays involving penguin amplitudes. A U-spin analysis of the time-dependent $C P$ asymmetries in the decays $B_{d} \rightarrow \pi^{+} \pi^{-}$ and $B_{s} \rightarrow K^{-} K^{+}$is expected to give a total uncertainty on $\gamma$ of $\simeq 7^{\circ}$ with one nominal year of data. A similar sensitivity is achievable via a Dalitz analysis of $B^{+} \rightarrow K^{+} \pi^{+} \pi^{-}$and $B_{d} \rightarrow K_{S} \pi^{+} \pi^{-}$. With relatively small amounts of data, discoveries of the rare modes $B_{d} \rightarrow K^{+} K^{-}, B_{s} \rightarrow \pi^{+} \pi^{-}$ and $B_{d} \rightarrow p \bar{p}$ are expected. The ultra-rare modes $B^{+} \rightarrow K^{-} \pi^{+} \pi^{+}$and $B^{+} \rightarrow K^{+} K^{+} \pi^{-}$may be observed if their branching ratios are significantly enhanced by NP effects.

12th International Conference on B-Physics at Hadron Machines

September 7-11, 2009

Heidelberg, Germany.

\footnotetext{
*Speaker.

${ }^{\dagger}$ on behalf of the LHCb collaboration.
} 


\section{The LHCb Experiment}

The LHCb experiment [1] is a dedicated heavy flavour physics experiment operating at the CERN Large Hadron Collider. It aims to carry out stringent tests of the Standard Model and probe New Physics effects by making precision measurements in the $B$ and $D$ sectors. This report describes the potential of $\mathrm{LHCb}$ to make such measurements using decays of $B$ hadrons to charmless hadronic final states.

LHCb is well suited to study hadronic $B$ decays, due to the following characteristics:

The Vertex Locator (VELO): a silicon strip detector placed close to the interaction point. The VELO makes precise measurements of the positions of primary and secondary vertices, which allows $B$ decays to be distinguished from background events, and also gives excellent resolution ( $\simeq 40 \mathrm{fs}$ ) on the $B$ hadron proper time. This resolution makes time-dependent studies of $B_{s}$ decays possible.

Ring Imaging Cherenkov (RICH) counters: these provide excellent particle identification (PID) capability for protons, pions and kaons in the momentum range $1-100 \mathrm{GeV} / \mathrm{c}$. This allows different hadronic final states to be separated from each other.

Trigger strategy: information from the VELO is used at an early stage of the trigger process to identify displaced vertices. Hadrons with high transverse momentum $\left(p_{T}\right)$ can be triggered on in the first stage of the trigger. These features allow good trigger efficiency for hadronic final states.

\section{Measurements of the CKM Parameter $\gamma$}

This section outlines two different methods by which $\mathrm{LHCb}$ aims to measure the CKM angle $\gamma$ using charmless hadronic $B$ decays.

Improving on current measurements of $\gamma$, the least well-constrained parameter of the "main" (db) unitarity triangle, is one of the key goals of the physics programme of the LHCb experiment. Performing a global CKM fit [2] using all the available information yields a reasonably tight constraint on $\gamma$ :

$$
\gamma_{\mathrm{CKM} \mathrm{fit}}=\left(67.9_{-3.7}^{+4.3}\right)^{\circ}
$$

with an error similar in size to those on the other CKM angles. However the constraint from direct measurements of $\gamma$ is far weaker [2]:

$$
\gamma_{\text {directmeas. }}=\left(75_{-22}^{+19}\right)^{\circ}
$$

LHCb will make direct measurements of $\gamma$ using two different classes of $B$ decay: those that proceed via tree amplitudes only, and those in which both tree and penguin amplitudes play a significant rôle. The aim is not only to tighten the constraint on $\gamma$, but also to search for inconsistencies in the measured values of $\gamma$ in different decays, which would reveal the presence of New Physics. Such inconsistencies can appear because the value of $\gamma$ measured from "tree-only" decays is unlikely to be affected by New Physics, as in most NP models the new particles do not enter at tree level. However for decays where penguin amplitudes play a significant rôle, new virtual particles can appear in the loop, affecting the penguin amplitude and thus the measured value of $\gamma$ for such decays. 
The two methods that are described below involve charmless hadronic $B$ decays that feature both tree and penguin amplitudes. A description of planned $\gamma$ measurements at LHCb using "treeonly" decays can be found in [3].

\subsection{Measuring $\gamma$ Using $B_{d} \rightarrow \pi^{+} \pi^{-}$and $B_{s} \rightarrow K^{-} K^{+}$}

A method was proposed by Fleischer in [4] to extract $\gamma$ from measurements of the direct and mixing-induced $C P$ asymmetries in the decays ${ }^{1} B_{d} \rightarrow \pi^{+} \pi^{-}$and $B_{s} \rightarrow K^{-} K^{+}$. The method is an attractive one because it does not neglect any of the different topologies contributing to each decay, and so does not rely on any model-dependent assumptions about their relative sizes. The only assumption made, using the fact that these two decays are exact U-spin conjugates of each other, is that the final-state strong interaction effects are the same for each decay. Violations of this assumption due to possible U-spin breaking effects are the only source of theoretical error in the method.

The direct and mixing-induced $C P$ asymmetries are measured in each channel using a tagged, time-dependent analysis:

$$
A_{C P}(t) \equiv \frac{\Gamma\left(N^{0} \rightarrow f\right)-\Gamma\left(\bar{N}^{0} \rightarrow f\right)}{\Gamma\left(N^{0} \rightarrow f\right)+\Gamma\left(\bar{N}^{0} \rightarrow f\right)}=\frac{A_{C P}^{\mathrm{dir}} \cos (\Delta m t)-A_{C P}^{\mathrm{mix}} \sin (\Delta m t)}{\cosh (\Delta \Gamma t / 2)-A_{\Delta \Gamma} \sinh (\Delta \Gamma t / 2)} .
$$

The four asymmetries can be shown [4] to depend on seven physics parameters:

$$
\begin{aligned}
& A_{\pi^{+} \pi^{-}}^{\mathrm{dir}}=f_{1}(d, \theta, \gamma), \quad A_{\pi^{+} \pi^{-}}^{\mathrm{mix}}=f_{2}\left(d, \theta, \gamma, \phi_{d}\right), \\
& A_{K^{+} K^{-}}^{\mathrm{dir}}=f_{3}\left(d^{\prime}, \theta^{\prime}, \gamma\right), \quad A_{K^{+} K^{-}}^{\mathrm{mix}}=f_{4}\left(d^{\prime}, \theta^{\prime}, \gamma, \phi_{s}\right),
\end{aligned}
$$

where $\phi_{d}$ and $\phi_{s}$ are the mixing phases of the $B_{d}$ and $B_{s}$ respectively. The quantities $d$ and $\theta$ parameterise respectively the magnitude and phase differences in the strong interaction dynamics between the tree and penguin contributions to the decay $B_{d} \rightarrow \pi^{+} \pi^{-}$, with $d^{\prime}$ and $\theta^{\prime}$ being the equivalent quantities for $B_{s} \rightarrow K^{-} K^{+}$. Under the assumption of U-spin symmetry, the two decays share the same strong dynamics, so that $d=d^{\prime}$ and $\theta=\theta^{\prime}$. It should be noted that even if U-spin symmetry is broken, the only corrections to these equalities will be non-factorisable corrections, which are expected to be small [4]. With these equalities, and taking the value of $\phi_{d}$ from the analysis of the $B_{d} \rightarrow J / \psi K_{S}$ decay, the number of unknowns in the system 2.4 is reduced from seven to four, so the system becomes soluble.

The steps involved in the analysis at LHCb of two-body charmless hadronic $B$ decays (referred to as $B \rightarrow h^{+} h^{\prime}$ - decays) are described in detail in [5], with an emphasis on the extraction of $\gamma$ from $B_{d} \rightarrow \pi^{+} \pi^{-}$and $B_{s} \rightarrow K^{-} K^{+}$. The main points of this analysis will now be summarised.

At LHCb, $B \rightarrow h^{+} h^{\prime}-$ events are selected by searching for two tracks that have high impact parameters with respect to the primary vertex, and high $p_{T}$. The $B$ candidate formed from these tracks should have a small impact parameter and large flight distance with respect to the primary vertex. After applying this selection, and also the trigger selection for hadronic decays, the expected yields of $B_{d} \rightarrow \pi^{+} \pi^{-}$and $B_{s} \rightarrow K^{-} K^{+}$events with $2 \mathrm{fb}^{-1}$ of LHCb data are 59000 and 72000 respectively.

\footnotetext{
${ }^{1}$ The inclusion of charge-conjugated modes is implied throughout this report.
} 


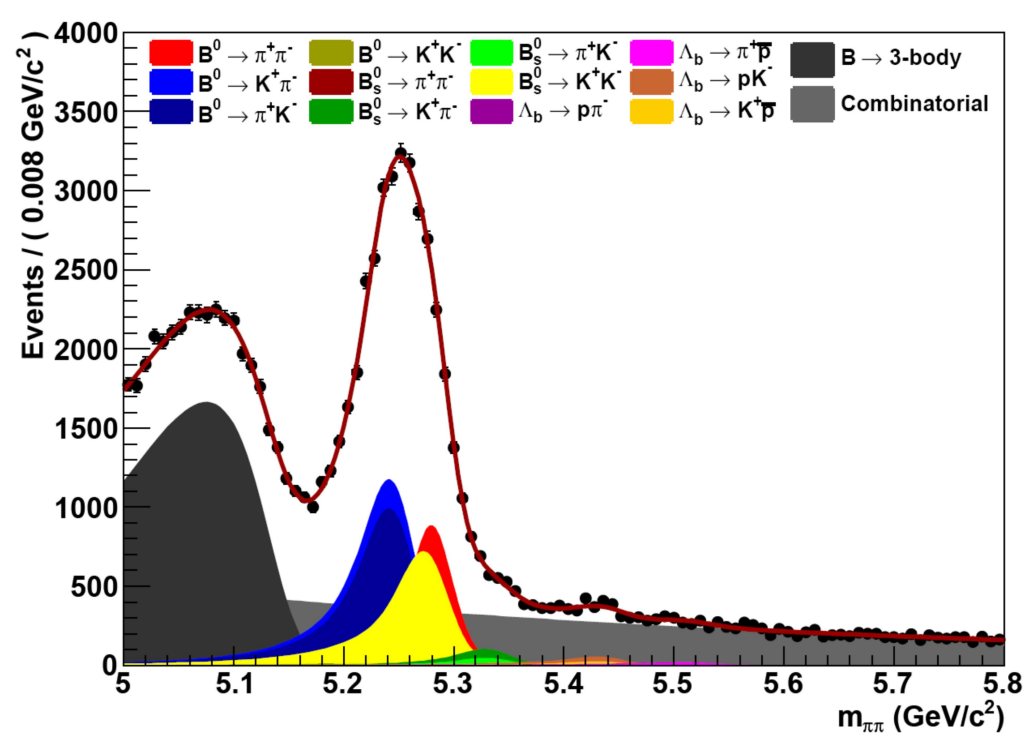

Figure 1: Invariant mass distributions for selected and triggered $B \rightarrow h^{+} h^{\prime}-$ signal and background events, expected at $\mathrm{LHCb}$ with $0.2 \mathrm{fb}^{-1}$ of data. The backgrounds considered are partially reconstructed three-body decays and combinatoric background.

A simultaneous fit to extract event yields, lifetimes and $C P$ asymmetries is performed to the $B \rightarrow h^{+} h^{\prime}$ - events, using the mass, proper time, flavour tagging and PID information. Each of these distributions needs to calibrated on data as accurately as possible, minimising the reliance on Monte Carlo simulations.

Mass: The invariant mass distributions for $B \rightarrow h^{+} h^{\prime}$ signal and background events are shown in Fig. 1. All events are reconstructed under the $\pi^{+} \pi^{-}$mass hypothesis. The resulting shift of the measured mass for each decay is an analytical function of the momentum asymmetry $\left(\left|p_{\pi^{+}}\right|-\left|p_{\pi^{-}}\right|\right) /\left(\left|p_{\pi^{+}}\right|+\left|p_{\pi^{-}}\right|\right)$. The radiative tail due to the emission of photons from the final state can be parameterised analytically.

Proper Time: For $B_{s}$ decays, where the oscillation frequency is high, knowledge of the proper time resolution is important. The resolution for $B_{s} \rightarrow K^{-} K^{+}$can be calibrated with the flavour-specific decay $B_{s} \rightarrow K^{-} \pi^{+}$. For both $B_{d}$ and $B_{s}$ decays, the selection cuts remove events with small proper times, biasing the lifetime distribution (although such a bias cancels out to first order when measuring the asymmetry). This effect can be quantified using the so-called "swimming" method $[6,7]$, which determines on an event-by-event basis the minimum lifetime required for an event to pass the selection.

Flavour Tagging: The percentage of events with incorrectly assigned flavour tag (mistag rate) for $B_{d}$ decays is measurable using the flavour-specific decay $B_{d} \rightarrow K^{+} \pi^{-}$. For $B_{s}$ decays the situation is more difficult as the equivalent channel $B_{s} \rightarrow K^{-} \pi^{+}$has a smaller yield and is also sensitive to the proper time resolution. Hence measuring the mistag rate for $B_{s}$ channels may require input from other $B_{s}$ decay modes, such as $B_{s} \rightarrow D_{s}^{-} \pi^{+}$.

Particle Identification: The PID likelihoods can be calibrated using specially-obtained samples of certain decays, where high-purity samples of each type of charged hadron can be selected using only kinematic cuts. The $K-\pi$ separation will be measured using $D^{*+} \rightarrow D^{0}(K \pi) \pi^{+}$decays, and 
the $p-\pi$ separation using $\Lambda \rightarrow p \pi^{-}$decays.

The uncertainties on the $C P$ asymmetries for $B_{d} \rightarrow \pi^{+} \pi^{-}$and $B_{s} \rightarrow K^{-} K^{+}$with $2 \mathrm{fb}^{-1}$ of data using the simultaneous fit are each around 0.04. The corresponding uncertainties on the physics parameters are then calculated, allowing for some breaking of U-spin symmetry (the constraints $0.8<d / d^{\prime}<1.2$ and $\left|\theta-\theta^{\prime}\right|<20^{\circ}$ are imposed). The uncertainties on $\gamma$ and $\phi_{s}$ are found to be $7^{\circ}$ and $0.06 \mathrm{rad}$ respectively. An alternative strategy to extract $\gamma$ would be to use the value of $\phi_{s}$ measured using the decay $B_{s} \rightarrow J / \psi \phi$, where an error of around $0.04 \mathrm{rad}$ is expected with $2 \mathrm{fb}^{-1}$ of data [8]. This would allow one of the U-spin constraints to be fully released.

\subsection{Measuring $\gamma$ Using $B^{+} \rightarrow K^{+} \pi^{+} \pi^{-}$and $B_{d} \rightarrow K_{S} \pi^{+} \pi^{-}$}

Another method to measure $\gamma$ using decays containing penguin amplitudes was proposed by Bediaga et al. in [9]. It uses a Dalitz analysis of the decays $B^{+} \rightarrow K^{+} \pi^{+} \pi^{-}$and $B_{d} \rightarrow K_{S} \pi^{+} \pi^{-}$, and will now be briefly described.

The method makes use of the fact that the two decays can proceed via the same intermediate resonances, and hence they have some common contributions to their amplitudes. The dominant penguin contribution to $B^{+} \rightarrow K^{+} \pi^{+} \pi^{-}$can be extracted via a standard Dalitz analysis, as the final state is self-tagging. This contribution is also present in $B_{d} \rightarrow K_{S} \pi^{+} \pi^{-}$, but an additional large tree amplitude, carrying a weak phase of $\gamma$, is also present. The measurement from $B^{+} \rightarrow K^{+} \pi^{+} \pi^{-}$is used as input to an untagged Dalitz analysis of $B_{d} \rightarrow K_{S} \pi^{+} \pi^{-}$to measure the tree amplitude, and hence extract $\gamma$. The Dalitz analysis for $B_{d} \rightarrow K_{S} \pi^{+} \pi^{-}$can be untagged because the resonance bands overlap in distinct areas for $B_{d}$ and $\overline{B_{d}}$, as is illustrated in Fig. 2.

The LHCb selection strategy for three-body charmless hadronic decays is similar to that for the two-body case, but with the additional requirement that the transverse momentum cuts should not be too tight, as this reduces the selection efficiency in the low-mass corner of the Dalitz plot.

The expected yields with $2 \mathrm{fb}^{-1}$ of data following the selection and the early stages of the trigger are around 500000 for $B^{+} \rightarrow K^{+} \pi^{+} \pi^{-}$and around 90000 for $B_{d} \rightarrow K_{S} \pi^{+} \pi^{-}$. The lower yield in the neutral mode is partly due to its lower branching ratio, and partly due to the lower reconstruction efficiency for $K_{S}$ (which may decay after leaving the VELO, where they are less likely to be reconstructed).

A toy simulation study, not including background or detector effects, gives an statistical uncertainty of around $5^{\circ}$ on $\gamma$ with $2 \mathrm{fb}^{-1}$ using this method [9]. Once the currently neglected effects are taken into account it is likely that the total sensitivity will be similar to that achievable with $B_{d} \rightarrow \pi^{+} \pi^{-}$and $B_{s} \rightarrow K^{-} K^{+}$.

\section{Rare Charmless Hadronic $B$ Decays}

In both the two-body and three-body sectors, there are charmless hadronic $B$ decay modes that are possible in the SM, but which have yet to be observed experimentally. A brief description of these modes, and the prospects of LHCb to make an observation of them, is given below.

\subsection{Two-body Rare Decays}

The decays $B_{d} \rightarrow K^{+} K^{-}$and $B_{s} \rightarrow \pi^{+} \pi^{-}$proceed in the SM only via annihilation and exchange diagrams; hence their SM branching ratios are small, $\mathscr{O}\left(10^{-6}\right)$ or less [10]. Recent mea- 


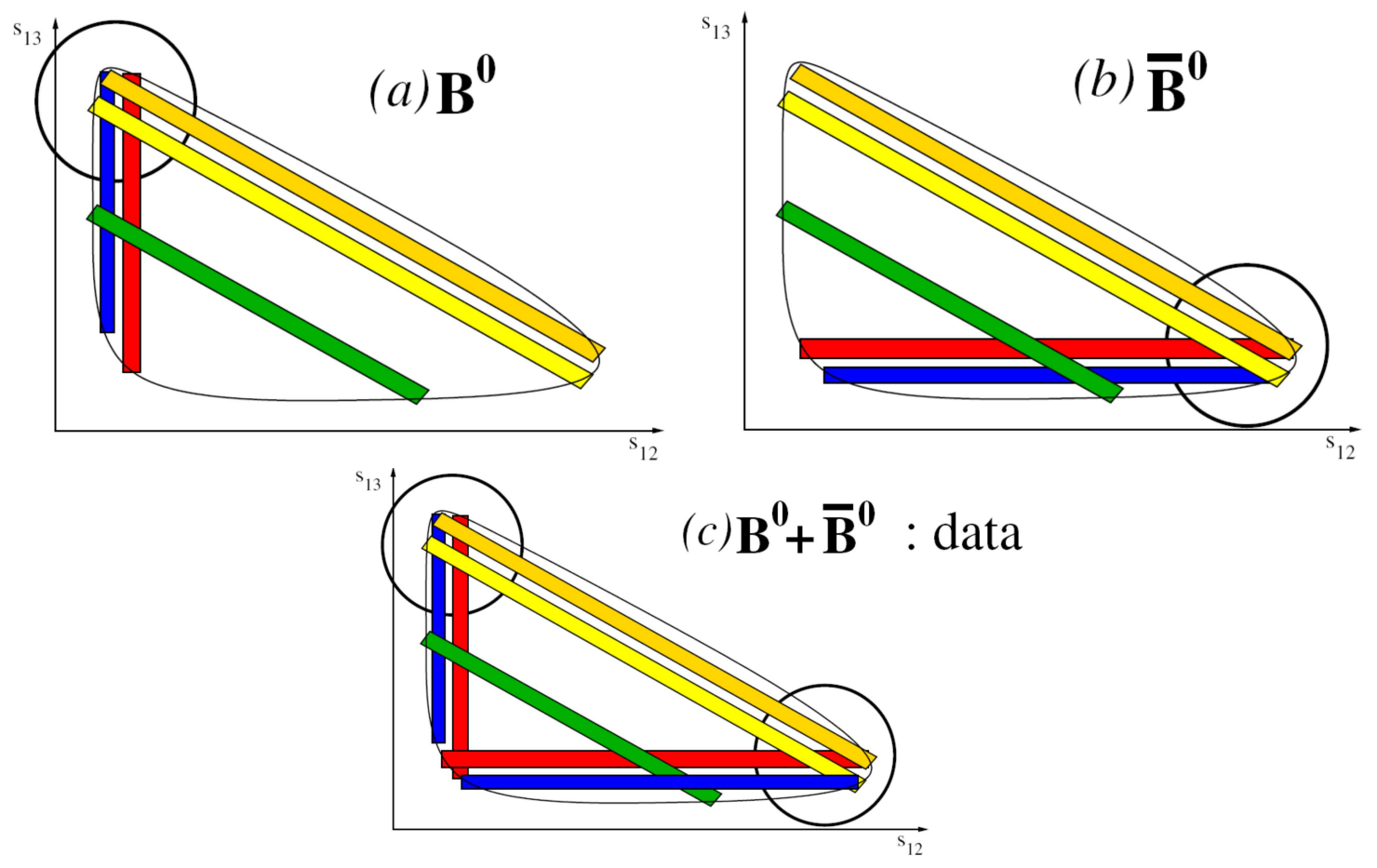

Figure 2: Schematics of Dalitz plots for the decays $\left\{B_{d}, \overline{B_{d}}\right\} \rightarrow K_{S} \pi^{+} \pi^{-}$. The horizontal (vertical) axes correspond to the $K_{S} \pi^{+}\left(K_{S} \pi^{-}\right)$squared masses respectively. The resonances shown are: $K^{*}(892)^{ \pm}$(blue), $K^{*}(1430)^{ \pm}$(red), $\rho(770)^{0}$ (orange), $f_{0}(980)$ (yellow) and $\chi_{c 0}$ (green). The fact that the resonance overlaps (circled) occur in distinct regions of the plot for $B_{d}$ and $\overline{B_{d}}$ obviates the need for tagging of the initial flavour.

surements of their branching ratios from the CDF experiment showed a small excess of events in both modes, and yielded the upper limits $\mathscr{B}\left(B_{d} \rightarrow K^{+} K^{-}\right)<0.7 \times 10^{-6}$ and $\mathscr{B}\left(B_{s} \rightarrow \pi^{+} \pi^{-}\right)<$ $1.2 \times 10^{-6}$, at $90 \%$ confidence level [11]. The excellent mass resolution and PID capabilities of LHCb will be crucial in separating these decays from the more abundant $B \rightarrow h^{+} h^{\prime}$ modes. LHCb expects to achieve $5 \sigma$ observations of both of these decays with around $1 \mathrm{fb}^{-1}$ of data, if their branching ratios are not significantly lower than the SM expectations [12].

Another unobserved two-body charmless hadronic $B$ decay that $\mathrm{LHCb}$ is well-suited to search for is the baryonic mode $B_{d} \rightarrow p \bar{p}$. No charmless two-body baryonic $B$ decay has yet been observed, and theoretical predictions of their branching ratios within the SM vary widely depending on the method of calculation used (for a review of theoretical predictions see [13]). This variation is mainly due to the difficulty of correctly describing the quark-antiquark pair production from the vacuum that is required to produce the two baryons in the final state.

The Belle experiment has placed the best current upper limit of $1.1 \times 10^{-7}$ on the $B_{d} \rightarrow p \bar{p}$ branching ratio [14]. This limit excludes most of the theoretical predictions, and any tightening of the limit will exclude the remaining predictions.

The main background to $B_{d} \rightarrow p \bar{p}$ at $\mathrm{LHCb}$ is expected to come from combinatorics, as specific backgrounds from two and three-body hadronic $B$ decays can be very efficiently suppressed using PID requirements [5].

Assuming that the true $B_{d} \rightarrow p \bar{p}$ branching ratio is close to the current experimental limit, the 
upper limit (at 90\% confidence level) on the expected background-to-signal (B/S) ratio has been calculated to be $\simeq 2.2$. Most of this background is from combinatorics. Conservatively taking this upper limit as the central value for the $\mathrm{B} / \mathrm{S}$ ratio, an observation of $B_{d} \rightarrow p \bar{p}$ can be made with around $0.25 \mathrm{fb}^{-1}$ of data at nominal centre-of-mass energy.

\subsection{Three-body Rare Decays}

The "wrong sign" three-body charmless hadronic $B$ decays $B^{+} \rightarrow K^{-} \pi^{+} \pi^{+}$and $B^{+} \rightarrow K^{+} K^{+} \pi^{-}$ (and their charge conjugates) are predicted to have tiny branching ratios in the SM; they can only proceed via convoluted processes involving both internal loops and gluon emission. The resulting SM predictions are of the orders of $10^{-14}$ and $10^{-11}$ for $B^{+} \rightarrow K^{-} \pi^{+} \pi^{+}$and $B^{+} \rightarrow K^{+} K^{+} \pi^{-}$ respectively.

However, their branching ratios can be strongly enhanced in certain New Physics models. For example in models with a new heavy vector boson $Z^{\prime}$, the $B^{+} \rightarrow K^{-} \pi^{+} \pi^{+}$branching ratio can be as high as $10^{-6}$, which is close to the current limit from experiment. If such NP enhancements are present, $\mathrm{LHCb}$ may be able to make an observation of these three-body decays.

\section{Summary}

The LHCb experiment has a rich physics programme involving charmless hadronic $B$ decays. The CKM angle $\gamma$ can be measured to an accuracy of $7^{\circ}$ with one nominal year of data, using a time-dependent analysis of the $C P$ asymmetries in the decays $B_{d} \rightarrow \pi^{+} \pi^{-}$and $B_{s} \rightarrow K^{-} K^{+}$. A similar sensitivity should be achievable with a Dalitz analysis of the decays $B^{+} \rightarrow K^{+} \pi^{+} \pi^{-}$and $B_{d} \rightarrow K_{S} \pi^{+} \pi^{-}$. The $\gamma$ values measured in these analyses can be compared to those found in decays without penguin contributions. Any significant discrepancy would be a sign of New Physics in loop diagrams.

LHCb can also make the first discoveries of some rare charmless hadronic $B$ decays. Observations of $B_{d} \rightarrow K^{+} K^{-}, B_{s} \rightarrow \pi^{+} \pi^{-}$and $B_{d} \rightarrow p \bar{p}$ are all expected with less than one nominal year of data. The decays $B^{+} \rightarrow K^{-} \pi^{+} \pi^{+}$and $B^{+} \rightarrow K^{+} K^{+} \pi^{-}$may be seen if their branching ratios are significantly enhanced by New Physics.

\section{References}

[1] A. Augusto Alves Jr et al. (LHCb Collaboration), JINST 03 S08005 (2008).

[2] J. Charles et al. (CKMFitter Group), Eur. Phys. J. C 411 (2005). Updated results and plots available at: http://ckmfitter.in2p3.fr.

[3] S. Ricciardi, these proceedings.

[4] R. Fleischer, Phys. Lett. B 459306 (1999).

[5] A. Bates et al., CERN-LHCb-ROADMAP6, to be published.

[6] F. Azfar et al., CDF-6756 (2003).

[7] V. Gligorov and J. Rademacker, CERN-LHCb-2007-053 (2007).

[8] O. Leroy, these proceedings. 
[9] I. Bediaga et al., Phys. Rev. D 76073011 (2007).

[10] M. Gronau et al., Phys. Rev. D 526356 (1995)

[11] T. Aaltonen et al. (CDF Collaboration), Phys. Rev. Lett. 103031801 (2009). See also D. Tonelli, these proceedings.

[12] C. Newby, PhD Thesis, University of Oxford (2005). CERN-THESIS-2007-018.

[13] H. Y. Cheng and K. C. Yang, Phys. Rev. D 66014020 (2002).

[14] Y. T. Tsai et al. (Belle Collaboration), Phys. Rev. D 75111101 (2007). 